Original Article

\title{
Gender-differences in disease distribution and outcome in hospitalized elderly: Data from the REPOSI study
}

\author{
S. Corrao ${ }^{\mathrm{a}, \mathrm{b}}$, P. Santalucia c,d,*, C. Argano a ${ }^{\mathrm{a}}$, C.D. Djade ${ }^{\mathrm{c}, \mathrm{d}}$, E. Barone ${ }^{\mathrm{a}}$, M. Tettamanti ${ }^{\mathrm{d}}$, L. Pasina ${ }^{\text {d }}$, C. Franchi ${ }^{\mathrm{d}}$, \\ T. Kamal Eldin ${ }^{\mathrm{d}}$, A. Marengoni ${ }^{\mathrm{e}}$, F. Salerno ${ }^{\mathrm{f}}$, M. Marcucci ${ }^{\mathrm{d}, \mathrm{g}}$, P.M. Mannucci ${ }^{\mathrm{c}}$, A. Nobili ${ }^{\mathrm{d}}$ and REPOSI Investigators ${ }^{1}$ \\ a Dipartimento Biomedico di Medicina Interna e Specialistica (DiBiMIS), University of Palermo, Italy \\ b Department of Internal Medicine 2, National Relevance Hospital Trust, ARNAS Civico, Di Cristina e Benfratelli, Palermo, Italy \\ c Scientific Direction, IRCCS Foundation Maggiore Hospital Policlinico, Milan, Italy \\ d Department of Neuroscience, IRCCS Istituto di Ricerche Farmacologiche Mario Negri, Milan, Italy \\ e Department of Clinical and Experimental Science, University of Brescia, Italy \\ ${ }^{\mathrm{f}}$ Medicina Interna, IRCCS Policlinico San Donato, Department of Medical and Surgery Sciences, University of Milano, Italy \\ ${ }^{g}$ Department of Internal Medicine, IRCCS Ca' Granda Maggiore Policlinico Hospital Foundation, Milano, Italy
}

\section{A R T I C L E I N F O}

\section{Article history:}

Received 19 April 2014

Received in revised form 16 June 2014

Accepted 26 June 2014

Available online 19 July 2014

\section{Keywords:}

Elderly

Sex-gender difference

In-hospital mortality

Disease distribution

\begin{abstract}
A B S T R A C T
Background and purpose: Women live longer and outnumber men. On the other hand, older women develop more chronic diseases and conditions such as arthritis, osteoporosis and depression, leading to a greater number of years of living with disabilities. The aim of this study was to describe whether or not there are gender differences in the demographic profile, disease distribution and outcome in a population of hospitalized elderly people.

Methods: Retrospective observational study including all patients recruited for the REPOSI study in the year 2010. Analyses are referred to the whole group and gender categorization was applied.

Results: A total of 1380 hospitalized elderly subjects, $50.5 \%$ women and $49.5 \%$ men, were considered. Women were older than men, more often widow and living alone or in nursing homes. Disease distribution showed that malignancy, diabetes, coronary artery disease, chronic kidney disease and chronic obstructive pulmonary disease were more frequent in men, but hypertension, osteoarthritis, anemia and depression were more frequent in women. Severity and comorbidity indexes according to the Cumulative Illness Rating Scale (CIRS-s and CIRS-c) were higher in men, while cognitive impairment evaluated by the Short Blessed Test (SBT), mood disorders by the Geriatric Depression Scale (GDS) and disability in daily life measured by Barthel Index (BI) were worse in women. In-hospital and 3-month mortality rates were higher in men.

Conclusions: Our study showed a gender dimorphism in the demographic and morbidity profiles of hospitalized elderly people, emphasizing once more the need for a personalized process of healthcare.
\end{abstract}

(c) 2014 European Federation of Internal Medicine. Published by Elsevier B.V. All rights reserved.

\section{Introduction}

The percentage of people aged 60 years and over has gradually increased during the last decades due to the improvements in healthcare [1,2] and is expected to further increase up to nearly $30 \%$ of the European population by the year 2050. Women are the largest portion of elderly people reaching $64 \%$ of those 80 years or older [3]. Aging is associated with multiple chronic conditions [4], ranging in

\footnotetext{
* Corresponding author at: Fondazione IRCCS Ospedale Maggiore Policlinico, Medicina D'Urgenza, Padiglione Devoto, Via F. Sforza, 35, 20122 Milan, Italy. Tel.: + 3902 55034710; fax: + 390255033600 .

E-mail address: p_santalucia@hotmail.com (P. Santalucia).

1 REPOSI denotes the REgistro POliterapie SIMI, Società Italiana di Medicina Interna. The participating units and co-authors are listed in the Appendix.
}

prevalence between $55 \%$ and $98 \%$ in people over 65 years of age [5]. Female sex along with old age and low socio-economic level is associated with multi-morbidity and an increased risk of hospitalization [5-8].

In the general population, gender differences are known for cardiovascular disease in terms of age distribution and impact of risk factors, clinical presentation and outcome $[9,10]$. Moreover, in the aging population the female sex is more frequently associated with depression $[11,12]$ and cognitive disorders [13], that are likely to be responsible for physical function decline and poor quality of life $[14,15]$. However, only few data are available on gender differences in terms of disease distribution and outcome in elderly subjects admitted to hospital.

Hence, the Registro Politerapie SIMI (REPOSI) project [16], that was designed to investigate the impact of multi-morbidity and related polypharmacy in the elderly population admitted to internal medicine and geriatric wards in Italy, was thought to be an appropriate setting 
in order to investigate whether or not there is any gender difference in socio-demographic profile, clinical characteristics, disease distribution and outcome in the hospitalized elderly.

\section{Methods}

All patients recruited for the REPOSI study during the year 2010 have been included in this analysis and the available information were analyzed on the basis of a gender perspective. Socio-demographic variables such as age classes, education, marital status, work occupation, living arrangement and need for assistance in daily living, were all considered along with laboratory and clinical characteristics.

Disease distribution at hospital admission, including frequency of diagnoses higher than $10 \%$ was taken into account. The International Classification of Diseases-Ninth Revision (ICD-9) was used for disease classification [17]. Cognitive status and mood disorders, tested by the Short Blessed Test (SBT) [18] and Geriatric Depression Scale (GDS) [19], were considered. Functional status at hospital admission was measured by means of the Barthel Index (BI) [20] and classified as mild (BI 75-90), moderate (BI 50-74), severe (BI 25-49) and total dependence (BI 0-24). The severity and comorbidity indexes, evaluated respectively by the Cumulative Illness rating Scale (CIRS-S and CIRS-c) [21], were also included in the gender analysis. Finally gender-differences in outcome measures, such as length of hospital stay, destination at hospital discharge and in-hospital and 3 month-mortality rates were also considered.

\section{Statistical analysis}

Data are reported as percentages for categorical variables and as means ( $95 \%$ confidence intervals) for quantitative variables. Analyses are referred to the whole group and gender categorization was applied. A Barthel Index score $\leq 40$ was used to select patients with significant disability according to our population characteristics. The comparison between groups was made using the exact Fisher test for contingency tables and the $\mathrm{z}$ test for the comparison of proportions. The nonparametric Mann-Whitney $U$ test was used for the comparison of quantitative variables. Multivariate logistic analysis was used to explore the relationship between variables and outcomes (in-hospital and 3-month follow-up mortalities). Odds ratios (ORs) and 95\% confidence intervals (95\% CIs) were computed. The choice of variables was performed according to the Hosmer-Lemeshow methodology [24]: after univariate analysis, only variables with a $\mathrm{p}<0.20$ were included in the final model; then, through a backward process, variables were excluded until a significance level of $p<0.20$ was reached for each variable. A two-tailed $\mathrm{p}<0.05$ was considered statistically significant. Stata (StataCorp. 2011. Stata Statistical Software: Release 12. College Station, TX: StataCorp LP) was used for database management and analysis.

\section{Results}

We analyzed data on 1380 hospitalized patients aged 65 years or older enrolled in the REPOSI study during the year 2010, both sexes being equally represented. Women were slightly older than men, more frequently widow and living alone or in nursing homes (Table 1).

The analyses of the clinical characteristics categorized by gender (Table 2) showed that systolic blood pressure, body temperature, leucocytes, platelets and serum cholesterol were higher in women, but hemoglobin, fasting glucose level and serum creatinine were higher in men. Men were more often overweight ( $41.8 \%$ vs $30.5 \%$ ), although visceral obesity ( $>102 \mathrm{~cm}$ in men and $>88 \mathrm{~cm}$ in women) was more prevalent in women (62.2\% vs $29.6 \%)$.

The cumulative illness rating scale for the evaluation of severity and comorbidity indexes (CIRS-s and CIRS-c) were more frequently abnormal in men, while the SBT, GDS and BI were worse in women
(Table 2). Disease distribution showed that malignancy, diabetes, coronary artery disease, chronic kidney disease and chronic obstructive pulmonary disease were more frequent in men, while hypertension, osteoarthritis, anemia and depression were so in women (Table 3 ). Table 4 shows that in women there was a trend for a longer hospital stay than in men, however men had a higher in-hospital and 3-month mortalities. At multivariate analysis, both in-hospital and 3-month mortalities, the latter being statistically significant, were higher in men (Tables 5 and 6).

\section{Discussion}

This study from the REPOSI cohort of hospitalized elderly patients admitted acutely to internal or geriatric wards has shown the existence of gender differences in terms of demographic profile, clinical characteristics and outcomes. Although the attention of the scientific community on gender-related issues is high [22], there is little evidence specifically stemming from hospitalized patients with multiple diseases admitted to internal medicine and geriatric wards. Our observation that clinical profiles and disease distributions varied according to gender would support the existence of a gender dimorphism in the elderly. Women were older than men, consistently with epidemiological data on aging population [3], they were more often living alone due to their widowhood and longer life expectancy [23]. In REPOSI, women had a worse GDS score than men, confirming that there is a gender difference in the self-reporting assessment of symptoms related to depression.

Living alone is likely to be strictly related to depression that has been linked to several adverse health outcomes [25]. For instance, feeling lonely has been associated with higher age-related increases of systolic blood pressure [26] and an increased risk of incident coronary heart disease [27]. Attention has been recently devoted to the burden of mood disorders such as depression on cardiovascular disease [28-31], diabetes [32], hypertension [26] and stroke [33-35]. Furthermore, data from the Nurses Health Study showed an association between depression and cardiac events in women, namely sudden cardiac death and coronary artery disease [36]. A further investigation on the same cohort of 80,574 women aged between 54 and 79 years showed that a history of depression and the use of antidepressant drugs were associated with an increased risk for stroke [33]. Finally, INTERSTROKE showed that self-reported depression is associated with an increased risk of stroke (OR 1.35; 99\% CI 1.10-1.66) in 3000 cases and 3000 matched controls from 22 countries [37]. In elderly women the finding of a high prevalence of depression is often regarded as a marker of subclinical cerebrovascular disease [38], making them unsuitable for anti-depressant medication (particularly for selective serotonin reuptake inhibitors), that is associated with an increased risk of sudden death [39] and stroke [40].

Cognitive impairment tends to be more pronounced in elderly women than men [13], as also confirmed by the present analysis that showed that women scored worse at the SBT. Nahid and colleagues [41] found an increased risk of cognitive impairment and Alzheimer's disease in the female gender. Moreover, the PLUPAR study [42] showed that permanent cognitive impairment was more frequent in women.

Both depression and cognitive impairment may contribute to limitations in daily life activities, leading to a progressive deterioration of functional ability [43]. Our analysis showed that women had a worse functional status than men according to BI, that would be certainly related to the aging itself and therefore to the older age of women than men in our cohort, however it could also be the effect of a possible link between daily living activities, psychological and cognitive statuses. Data from the Women's Health Initiative Observational Study, conducted on a population of 61,609 women aged 50-79 years at baseline and followed up for 12 years, showed that those with a more sedentary life style reported a greater overall number of chronic diseases, a higher frequency of falls and disability in the activities of daily living, and that 
Table 1

Socio-demographic characteristics and risk factors of the REPOSI population.

\begin{tabular}{|c|c|c|c|c|}
\hline Variables & All patients & Women & Men & $\mathrm{p}$ \\
\hline $\mathrm{N}$ of subjects & 1380 & $697(50.5 \%)$ & $683(49.5 \%)$ & \\
\hline Age $^{\mathrm{a}}$ & $79(78.1-79.4)$ & $80.1(79.6-80.7)$ & $77.8(77.3-78.4)$ & $<0.0001$ \\
\hline Marital status (\%) & & & & $<0.001$ \\
\hline Married & 53.4 & 33.3 & 74.0 & \\
\hline Widow & 37.6 & 57.6 & 17.2 & \\
\hline Separated & 0.8 & 0.7 & 0.8 & \\
\hline Divorced & 0.9 & 0.7 & 1.1 & \\
\hline Single & 7.3 & 7.7 & 6.9 & \\
\hline Profession (\%) & & & & $<0.001$ \\
\hline Businessman & 1.9 & 0.4 & 3.5 & \\
\hline Manager & 12.7 & 9.8 & 15.8 & \\
\hline Tradesman & 5.4 & 5.1 & 5.8 & \\
\hline Other & 80.0 & 84.7 & 74.9 & \\
\hline Living arrangement (\%) & & & & $<0.001$ \\
\hline Alone & 23.5 & 32.7 & 14.3 & \\
\hline Spouse & 42.3 & 24.3 & 60.3 & \\
\hline Sons & 16.0 & 25.0 & 7.0 & \\
\hline Spouse and sons & 9.3 & 6.1 & 12.6 & \\
\hline Other & 8.9 & 11.9 & 5.8 & \\
\hline Institutionalized (\%) & 7.2 & 7.5 & 7.0 & 0.68 \\
\hline Caregiver (\%) & 56.5 & 59.2 & 53.7 & 0.03 \\
\hline Spouse & 31.5 & 11.6 & 53.7 & $<0.001$ \\
\hline Son & 51.4 & 67.0 & 34.0 & \\
\hline Other & 17.1 & 21.4 & 12.3 & \\
\hline \multicolumn{5}{|l|}{ Smoking habits (\%) } \\
\hline Smokers & 40.1 & 17.8 & 62.8 & $<0.001$ \\
\hline Former smokers & 8.9 & 5.2 & 12.8 & \\
\hline No smokers & 51.0 & 77.0 & 24.4 & \\
\hline Alcohol (\%) & 42.3 & 26.4 & 58.6 & $<0.001$ \\
\hline Need for urinary catheter (\%) & 23.3 & 23.7 & 23.0 & 0.75 \\
\hline \multicolumn{5}{|l|}{ Previous hospital admissions (\%) } \\
\hline No previous admission & 61.5 & 63.8 & 59.1 & 0.10 \\
\hline One previous admission & 25.7 & 23.7 & 27.8 & \\
\hline$>1$ previous admission & 12.8 & 12.5 & 13.1 & \\
\hline $\mathrm{N}^{\circ}$ previous admissions ${ }^{\mathrm{a}}$ & $5.7(5.3-6.2)$ & $5.4(4.8-6)$ & $6(5.4-6.8)$ & 0.11 \\
\hline
\end{tabular}

a Data are reported as mean (confidence interval 95\%).

they were more likely to live alone [44]. On the other hand, the present data show that men were more impaired than women in terms of cumulative illness burden with respect to severity and comorbidity (CIRS-S and CIRS-c).

Disease distribution favored a profile of a male subject that in his old age is affected more frequently than women by chronic obstructive pulmonary and coronary artery diseases and malignancies that are likely to be responsible for recurrent hospital admissions and higher in-hospital and short-term mortalities following hospitalization, yet without impairing their ability in daily life. Disease frequency, on the male side, is obviously associated to the gender differences in risk factor distribution, indeed men are more often smokers and former smokers, where tobacco smoke plays a well recognized role in determining each one of the mentioned diseases. On the female side, disease distribution showed a prevalence of such chronic conditions as osteoarthritis and anemia that are responsible for sedentary life and discomfort [45] and contribute to the identification of a female profile that in her old age is characterized by impairment in daily life activities even if without dominant severe diseases.

All in all, our findings are consistent with previous epidemiological studies pertaining to gender differences in terms of cognitive impairment, depression, quality of life and disability [44]. The greatest agreement with the literature concerns the demographic profile of the elderly according to gender [46], so that a higher percentage of men than women live with their spouse, who take on the role of caregiver despite their old ages. On the other hand, elderly women are more likely to rely for support upon non-spousal caregivers, such as their children or non-family members. Recently the PROFUND project has shown that having a caregiver different of spouse is a prognostic factor for increase of mortality [47]. In our cohort there isn't a statistical association between the caregiver and in-hospital or 3-month mortality Another major issue for elderly people is quality of life, that is worse in women than in men, perhaps owing to gender differences in living conditions, higher prevalence of chronic inflammatory diseases, pain and depression [14], conditions that usually deteriorate following an acute event causing hospitalization [48].

Our study has limitations. It is based upon data stemming from a general clinical evaluation for cognitive and affective disorders, based upon tools that are reliable for disease screening but not diagnostically conclusive. The study also lacks a lot of clinical information that was not collected by the minimum dataset of the REPOSI study; nor did we investigate whether or not there is an association between depression and cognitive impairment and specific medical therapies prescribed in hospital. However, in comparison to previous data on gender differences regarding risk factors and disease distribution that did mainly stem from population-based observational studies, this study should be considered an accurate picture of the situation of the elderly acutely hospitalized in internal medicine and geriatric wards. It also represents a reliable sample of the condition of elderly people with multi-morbidity that could be translated to a gender-difference analysis.

In conclusion, this study clearly shows a gender dimorphism in the demographic and morbidity profiles of hospitalized elderly people, women being older than men and more often affected by chronic conditions impairing their ability of daily life, while men did show more multimorbidity and higher short-term mortality after 
Table 2

Clinical characteristics of the REPOSI population at hospital admission.

\begin{tabular}{|c|c|c|c|c|}
\hline & All patients $(\mathrm{n}=1380)$ & $\begin{array}{l}\text { Women } \\
(\mathrm{n}=697)\end{array}$ & $\begin{array}{l}\text { Men } \\
(\mathrm{n}=683)\end{array}$ & $\mathrm{p}$ \\
\hline Systolic blood pressure $(\mathrm{mm} \mathrm{Hg})^{\mathrm{a}}$ & $133(132-134)$ & $134(133-136)$ & $132(131-134)$ & 0.04 \\
\hline Diastolic blood pressure $(\mathrm{mm} \mathrm{Hg})^{\mathrm{a}}$ & $75.4(74.8-76)$ & $75.8(74.9-76.7)$ & $75(74-76)$ & 0.32 \\
\hline Heart rate $(\mathrm{bpm})^{\mathrm{a}}$ & $80(79-81)$ & $83(81.5-84)$ & $79(77.6-80)$ & $<0.0001$ \\
\hline Body temperature $(\mathrm{mm} \mathrm{Hg})^{\mathrm{a}}$ & $36.8(36.3-37.2)$ & $37(36-37.9)$ & $36.4(36.4-36.5)$ & 0.03 \\
\hline Fasting glucose $(\mathrm{mg} / \mathrm{dL})^{\mathrm{a}}$ & $128(124-132)$ & $126(121-131)$ & $129(123-135)$ & 0.78 \\
\hline Creatinine $(\mathrm{mg} / \mathrm{dL})^{\mathrm{a}}$ & $1.2(1.2-1.3)$ & $1.12(1-1.18)$ & $1.38(1.3-1.46)$ & $<0.0001$ \\
\hline Hemoglobin $(\mathrm{mg} / \mathrm{dL})^{\mathrm{a}}$ & $11.9(11.8-12)$ & $11.6(11.5-11.8)$ & $12.2(12-12.4)$ & $<0.0001$ \\
\hline Leucocytes (cells per microliter) ${ }^{a}$ & $9155(8750-9561)$ & $9300(8640-9950)$ & $9000(8533-9482)$ & 0.55 \\
\hline Platelets (cells per microliter) ${ }^{\mathrm{a}}$ & $\begin{array}{l}228,000 \\
(223,000-233,000)\end{array}$ & $\begin{array}{l}242,500 \\
(235,000-250,000)\end{array}$ & $\begin{array}{l}214,000 \\
(206,000-221,000)\end{array}$ & $<0.0001$ \\
\hline Cholesterol (mg/dL) ${ }^{\mathrm{a}}$ & $163(160-167)$ & $171(167-175)$ & $154(151-158)$ & $<0.0001$ \\
\hline $\mathrm{BMI}^{\mathrm{a}}$ & $25.9(25.7-26.3)$ & $26.4(26-26.8)$ & $25.6(25.2-26)$ & 0.06 \\
\hline Waist circumference $(\mathrm{cm})^{\mathrm{a}}$ & $93.7(92.8-94.6)$ & $91.7(90.4-93)$ & $95.6(94.5-96.8)$ & $<0.0001$ \\
\hline Visceral obesity (\%) & 45.9 & 62.2 & 29.6 & $<0.0001$ \\
\hline Underweight patients (\%) & 3.9 & 4.6 & 3.1 & 0.17 \\
\hline Optimal weight patients (\%) & 43.3 & 41.7 & 43 & 0.64 \\
\hline Overweight patients (\%) & 36.1 & 30.5 & 41.8 & $<0.0001$ \\
\hline Obesity I (\%) & 12.3 & 15.6 & 9.0 & 0.0002 \\
\hline Obesity II (\%) & 3.6 & 5.2 & 1.9 & 0.0015 \\
\hline Obesity III (\%) & 1.8 & 2.4 & 1.2 & 0.10 \\
\hline Short Blessed Test score ${ }^{a}$ & $9.8(9.5-10.3)$ & $10.8(10.2-11.4)$ & $8.9(8.3-9.5)$ & 0.0001 \\
\hline Short Blessed Test score $\geq 10(\%)$ & 47.6 & 51.8 & 43.2 & 0.01 \\
\hline Barthel Index & $76.7(75.1-78.4)$ & $72.9(70.4-75.4)$ & $80.7(78.6-82.8)$ & $<0.0001$ \\
\hline Barthel Index $\leq 40(\%)$ & 16 & 20 & 12 & 0.0001 \\
\hline Geriatric depression scale $^{\mathrm{a}}$ & $1.3(1.2-1.4)$ & $1.4(1.3-1.5)$ & $1.2(1.1-1.3)$ & 0.002 \\
\hline $\mathrm{N}^{\circ}$ of drugs at hospital admission ${ }^{\mathrm{a}}$ & $5.4(5.3-5.6)$ & $5.2(5-5.4)$ & $5.6(5.4-5.9)$ & 0.01 \\
\hline $\mathrm{N}^{\circ}$ of in-hospital drugs $\mathrm{s}^{\mathrm{a}}$ & $4.0(3.9-4.4)$ & $4.0(3.7-4.3)$ & $4.2(3.9-4.5)$ & 0.38 \\
\hline $\mathrm{N}^{\circ}$ of drugs at hospital discharge $\mathrm{a}^{\mathrm{a}}$ & $6.4(6.2-6.6)$ & $6.3(6-6.5)$ & $6.6(6.3-6.8)$ & 0.08 \\
\hline Severity index (CIRS) ${ }^{a}$ & $1.6(1.63-1.66)$ & $1.61(1.6-1.7)$ & $1.7(1.6-1.7)$ & 0.0002 \\
\hline Comorbidity index $(\mathrm{CIRS})^{\mathrm{a}}$ & $2.9(2.8-3)$ & $2.7(2.6-2.9)$ & $3.0(2.9-3.2)$ & 0.0012 \\
\hline Comorbidity index $(\mathrm{CIRS})<3$ & $44.1 \%$ & $48.1 \%$ & $40 \%$ & 0.002 \\
\hline Comorbidity index (CIRS) $\geq 3$ & $55.9 \%$ & $51.9 \%$ & $60 \%$ & \\
\hline
\end{tabular}

${ }^{a}$ Data are reported as means (95\% confidence interval). BMI = Body Mass Index; underweight: BMI < 18.5; optimal weight: BMI: 18.5 to 24.9; overweight: BMI: 25 to 29.9; obesity I: BMI: 30 to 34.9; obesity II: BMI: 35 to 39.9 and obesity III: BMI $\geq 40$. CIRS = cumulative illness rating scale.

hospitalization. The awareness of a gender dualism in living conditions and disease distribution in the elderly may explain gender differences in outcomes and represent a starting point for a process of personalized care to elderly people in order to improve their quality of life.

\section{Learning points}

- Hospitalized elderly people show a gender dimorphism in the demographic and morbidity profiles.
- Disease distribution in hospitalized elderly shows that men are more frequently affected by COPD and CHD, while women present more often chronic conditions such as ostheoarthritis and anemia.

- Depression and cognitive impairment along with an impaired functional ability in daily life are more frequent in women and old women

\section{Conflict of interest statement}

All the authors have no conflicts of interest to declare.

Table 3

Most frequent diagnoses (frequency more than 10\%) in the REPOSI population and according to gender at hospital admission.

\begin{tabular}{|c|c|c|c|c|}
\hline & All patients $(\mathrm{n}=1380)$ & $\begin{array}{l}\text { Women } \\
(\mathrm{n}=697)\end{array}$ & $\begin{array}{l}\text { Men } \\
(\mathrm{n}=683)\end{array}$ & $\mathrm{p}$ \\
\hline Hypertension (\%) & 78.7 & 80.7 & 76.8 & 0.05 \\
\hline Malignancy (\%) & 29.0 & 21.0 & 37.2 & $<0.0001$ \\
\hline Peripheral vascular disease (\%) & 28.7 & 28.7 & 28.8 & 0.19 \\
\hline Diabetes mellitus (\%) & 28.6 & 24.2 & 32.8 & 0.0004 \\
\hline Coronary artery disease (\%) & 24.7 & 19.3 & 30.1 & $<0.0001$ \\
\hline Heart failure (\%) & 22.0 & 21.0 & 23.0 & 0.39 \\
\hline Chronic kidney disease (\%) & 21.6 & 15.7 & 27.6 & $<0.0001$ \\
\hline Atrial fibrillation (\%) & 21.0 & 21.2 & 20.7 & 0.83 \\
\hline Chronic obstructive pulmonary disease (\%) & 20.2 & 16.0 & 24.4 & 0.0001 \\
\hline Gastric disease (\%) & 17.7 & 18.2 & 17.2 & 0.64 \\
\hline Hypertensive cardiopathy (\%) & 15.0 & 15.7 & 14.3 & 0.45 \\
\hline Osteoarthritis (\%) & 13.5 & 18.4 & 8.5 & $<0.0001$ \\
\hline Depression (\%) & 12.6 & 14.7 & 10.4 & 0.01 \\
\hline Anemia (\%) & 12.1 & 13.8 & 10.4 & 0.05 \\
\hline Cardiac valvulopathy (\%) & 10.0 & 11.2 & 8.7 & 0.11 \\
\hline Diverticular disease (\%) & 10.0 & 9.1 & 11.0 & 0.25 \\
\hline Diverticular disease (\%) & 10.0 & 9.1 & 11.0 & 0.25 \\
\hline
\end{tabular}


Table 4

Length of hospital stay, destination at hospital discharge, in-hospital and 3-month mortalities of the whole REPOSI population according to gender.

\begin{tabular}{|c|c|c|c|c|}
\hline & All patients $(n=1380)$ & $\begin{array}{l}\text { Women } \\
(\mathrm{n}=697)\end{array}$ & $\begin{array}{l}\text { Men } \\
(\mathrm{n}=683)\end{array}$ & $\mathrm{p}$ \\
\hline Length of hospital stay ${ }^{\mathrm{a}}$ (days) & $11.2(10.5-11.8)$ & $11.5(10.5-12.5)$ & $10.8(9.9-11.9)$ & 0.37 \\
\hline In hospital mortality (\%) & 5.0 & 4.4 & 5.6 & 0.34 \\
\hline 3-Month mortality (\%) & 5.0 & 6.8 & 11.5 & 0.01 \\
\hline Destination at discharge & & & & 0.14 \\
\hline Home (\%) & 89.6 & 25.9 & 26.8 & \\
\hline Home care (\%) & 3.2 & 0.6 & 1.3 & \\
\hline Institution (\%) & 3.7 & 0.8 & 1.37 & \\
\hline
\end{tabular}

a Data are reported as means (95\% confidence interval).

\section{Appendix A}

Investigators and co-authors of the REPOSI (REgistro POliterapie SIMI, Società Italiana di Medicina Interna) Study Group are as follows:

Steering Committee: Pier Mannuccio Mannucci (Chair, Fondazione IRCCS Cà Granda Ospedale Maggiore Policlinico, Milano), Alessandro Nobili (co-chair, IRCCS-Istituto di Ricerche Farmacologiche "Mario Negri", Milano), Mauro Tettamanti, Luca Pasina, Carlotta Franchi (IRCCS-Istituto di Ricerche Farmacologiche "Mario Negri", Milano), Francesco Salerno (IRCCS Policlinico San Donato Milanese, Milano), Salvatore Corrao (ARNAS Civico, Di Cristina, Benfratelli, DiBiMIS, Università di Palermo, Palermo), Alessandra Marengoni (Spedali Civili di Brescia, Brescia), Maura Marcucci (Dipartimento di Medicina Interna, Fondazione IRCCS Cà Granda Ospedale Maggiore Policlinico, Milano).

Clincal data monitoring and revision: Eleonora Sparacio, Stefania Alborghetti, Rosa Di Costanzo (IRCCS-Istituto di Ricerche Farmacologiche "Mario Negri", Milano).

Database Management and Statistics: Mauro Tettamanti, Codjo Djignefa Djade (IRCCS-Istituto di Ricerche Farmacologiche "Mario Negri", Milano).

Investigators: Domenico Prisco, Elena Silvestri, Caterina Cenci, Tommaso Barnini (Azienda Ospedaliero Universitaria Careggi Firenze, SOD Patologia Medica); Giuseppe Delitala, Stefano Carta, Sebastiana Atzori (Azienda Mista Ospedaliera Universitaria, Sassari, Clinica Medica); Gianfranco Guarnieri, Michela Zanetti, Annalisa Spalluti (Azienda Ospedaliera Universitaria Ospedali Riuniti di Trieste, Trieste, Clinica Medica Generale e Terapia Medica); Maria Grazia Serra, Maria Antonietta Bleve (Azienda Ospedaliera "Cardinale Panico" di Tricase, Lecce, Unità Operativa Complessa Medicina); Massimo Vanoli, Giulia Grignani, Gianluca Casella (Azienda Ospedaliera della Provincia di Lecco, Ospedale di Merate, Lecco, Medicina Interna); Laura Gasbarrone (Azienda Ospedaliera Ospedale San Camillo Forlanini, Roma, Medicina Interna 1); Giorgio Maniscalco, Massimo Gunelli, Daniela Tirotta (Azienda Ospedaliera Ospedale San Salvatore, Pesaro, Soc Medicina Interna); Antonio Brucato, Silvia Ghidoni, Paola Di Corato (Azienda Ospedaliera Papa Giovanni XXIII, Bergamo, Medicina 1); Mauro Bernardi, Silvia Li Bassi, Luca Santi (Azienda Ospedaliera Policlinico Sant'Orsola-Malpighi, Bologna, Semeiotica Medica Bernardi); Giancarlo Agnelli, Alfonso Iorio, Maura Marcucci, Emanuela Marchesini (Azienda Ospedaliera Santa Maria della Misericordia, Perugia, Medicina Interna e Cardiovascolare); Elmo Mannarino, Graziana Lupattelli, Pamela Rondelli, Francesco Paciullo (Azienda Ospedaliera Santa Maria della Misericordia, Perugia, Medicina Interna, Angiologia, Malattie da Arteriosclerosi); Fabrizio Fabris, Michela Carlon, Francesca Turatto (Azienda Ospedaliera Università di Padova, Padova, Clinica Medica I); Maria Cristina Baroni, Marianna Zardo (Azienda Ospedaliera Università di Parma, Parma, Clinica e Terapia Medica); Roberto Manfredini,
Christian Molino, Marco Pala, Fabio Fabbian (Azienda OspedalieraUniversitaria Sant'Anna, Ferrara, Unità Operativa Clinica Medica); Ranuccio Nuti, Roberto Valenti, Martina Ruvio, Silvia Cappelli (Azienda Ospedaliera Università Senese, Siena, Medicina Interna I); Giuseppe Paolisso, Maria Rosaria Rizzo, Maria Teresa Laieta (Azienda Ospedaliera Universitaria della Seconda Università degli Studi di Napoli, Napoli, VI Divisione di Medicina Interna e Malattie Nutrizionali dell'Invecchiamento); Teresa Salvatore, Ferdinando Carlo Sasso (Azienda Ospedaliera Universitaria della Seconda Università degli Studi di Napoli, Napoli, Medicina Interna e Malattie Epato-Bilio Metaboliche Avanzate); Riccardo Utili, Emanuele Durante Mangoni, Daniela Pinto (Azienda Ospedaliera Universitaria della Seconda Università degli Studi di Napoli, Napoli, Medicina Infettivologica e dei trapianti); Oliviero Olivieri, Anna Maria Stanzial (Azienda Ospedaliera Universitaria Integrata di Verona, Verona, Unità Operativa di Medicina Interna B); Renato Fellin, Stefano Volpato, Sioulis Fotini (Azienda Ospedaliera Universitaria Ospedale Sant'Anna, Ferrara, Unità Operativa di Medicina Interna Gerontologia e Geriatria); Mario Barbagallo, Ligia Dominguez, Lidia Plances, Daniela D'Angelo (Azienda Ospedaliera Universitaria Policlinico Giaccone Policlinico di Palermo, Palermo, Unità Operativa di Geriatria e Lungodegenza); Giovanbattista Rini, Pasquale Mansueto, Ilenia Pepe (Azienda Ospedaliera Universitaria Policlinico P. Giaccone di Palermo, Palermo, Medicina Interna e Malattie Metaboliche); Giuseppe Licata, Luigi Calvo, Maria Valenti (Azienda Ospedaliera Universitaria Policlinico P. Giaccone di Palermo, Palermo, Medicina Interna e Cardioangiologia); Claudio Borghi, Enrico Strocchi, Elisa Rebecca Rinaldi (Azienda Ospedaliera Universitaria Policlinico S. Orsola-Malpighi, Bologna, Unità Operativa di Medicina Interna Borghi); Marco Zoli, Elisa Fabbri, Donatella Magalotti (Azienda Ospedaliera Universitaria Policlinico S. Orsola-Malpighi, Bologna, Unità Operativa di Medicina Interna Zoli); Alberto Auteri, Anna Laura Pasqui, Luca Puccetti (Azienda Ospedaliera Universitaria Senese, Siena, Medicina 3); Franco Laghi Pasini, Pier Leopoldo Capecchi, Maurizio Bicchi (Azienda Ospedaliera Universitaria Senese, Siena, Unità Operativa Complessa Medicina 2); Carlo Sabbà, Francesco Saverio Vella, Alessandro Marseglia, Chiara Valentina Luglio (Azienda Ospedaliero-Universitaria Consorziale Policlinico di Bari, Bari, Medicina Interna Universitaria C. Frugoni); Giuseppe Palasciano, Maria Ester Modeo, Annamaria Aquilino, Pallante Raffaele (Azienda Ospedaliero-Universitaria Consorziale Policlinico di Bari, Bari, Medicina Interna Ospedale "PendeFerrannini"); Stefania Pugliese, Caterina Capobianco (Azienda Ospedaliero-Universitaria Consorziale Policlinico di Bari, Bari, Clinica Medica I Augusto Murri); Alfredo Postiglione, Maria Rosaria Barbella, Francesco De Stefano (Azienda Ospedaliera Universitaria Policlinico Federico II di Napoli, Medicina Geriatrica Dipartimento di Clinica Medica); Luigi Fenoglio, Chiara Brignone, Christian Bracco, Alessia Giraudo (Azienda Sanitaria Ospedaliera Santa Croce e Carle di Cuneo, 
Table 5

Multivariate analysis of the association between variables and in-hospital mortality.

\begin{tabular}{llc}
\hline & Odds ratio (95\% C.I.) & $\mathrm{p}$ \\
\hline Age & $1.03(0.99-1.07)$ & 0.07 \\
Male gender & $1.68(0.95-2.97)$ & 0.07 \\
Barthel Index $\leq 40$ & $5.20(2.96-9.11)$ & $<0.0001$ \\
Severity index (CIRS) & $2.54(1.45-4.4)$ & 0.001 \\
Fasting blood glucose & $0.99(0.996-0.999)$ & 0.01 \\
Systolic blood pressure & $0.97(0.96-0.98)$ & $<0.0001$ \\
Malignancy & $1.46(0.83-2.57)$ & 0.18 \\
\hline
\end{tabular}

The model reports only variables with $\mathrm{p}<0.2$; see Statistical analysis Section.

Cuneo, S. C. Medicina Interna); Giuseppe Musca, Olga Cuccurullo (Azienda Sanitaria Provinciale di Cosenza Presidio Ospedaliero di Cetraro, Cosenza, Unità Operativa Complessa Medicina Interna); Luigi Cricco, Alessandra Fiorentini (СОВ Stabilimento Montefiascone, Viterbo, Unità Operativa Complessa di Geriatria e Medicina); Maria Domenica Cappellini, Giovanna Fabio, Sonia Seghezzi, Margherita Migone De Amicis (Fondazione IRCCS Cà Granda Ospedale Maggiore Policlinico, Milano, Unità Operativa Medicina Interna IA); Silvia Fargion, Paola Bonara, Mara Bulgheroni, Rosa Lombardi (Fondazione IRCCS Cà Granda Ospedale Maggiore Policlinico, Milano, Medicina Interna 1B); Fabio Magrini, Ferdinando Massari, Tatiana Tonella (Fondazione IRCCS Cà Granda Ospedale Maggiore Policlinico, Milano, Unità Operativa Medicina Cardiovascolare); Flora Peyvandi, Alberto Tedeschi, Raffaella Rossio (Fondazione IRCCS Cà Granda Ospedale Maggiore Policlinico, Milano, Medicina Interna 2); Guido Moreo, Barbara Ferrari, Luisa Roncari (Fondazione IRCCS Cà Granda Ospedale Maggiore Policlinico, Milano, Medicina Interna 3); Valter Monzani, Valeria Savojardo, Christian Folli, Maria Magnini (Fondazione IRCCS Cà Granda Ospedale Maggiore Policlinico, Milano, Medicina d'Urgenza); Daniela Mari, Paolo Dionigi Rossi, Sarah Damanti, Silvia Prolo (Fondazione IRCCS Cà Granda Ospedale Maggiore Policlinico, Milano, Geriatria); Maria Sole Lilleri (Fondazione IRCCS Cà Granda Ospedale Maggiore Policlinico, Milano, Medicina Generale ad Indirizzo Geriatrico); Luigi Cricco, Alessandra Fiorentini ( $С О B$ Viterbo, Stabilimento Montefiascone, Viterbo, UOC Geriatria e Medicina); Giuliana Micale (IRCCS Istituto Auxologico Italiano, Milano, Medicina Generale ad indirizzo Geriatrico); Mauro Podda, Carlo Selmi, Francesca Meda (IRCCS Istituto Clinico Humanitas, Milano, Clinica Medica); Francesco Salerno, Silvia Accordino, Alessio Conca, Valentina Monti (IRCCS Policlinico San Donato e Università di Milano, San Donato Milanese, Medicina Interna); Gino Roberto Corazza, Emanuela Miceli, Marco Vincenzo Lenti, Donatella Padula (IRCCS Policlinico San Matteo di Pavia, Pavia, Clinica Medica I, Reparto 11); Carlo L. Balduini, Giampiera Bertolino, Stella Provini, Federica Quaglia (IRCCS Policlinico San Matteo di Pavia, Pavia, Clinica Medica III); Giovanni Murialdo, Marta Bovio (IRCS Azienda Ospedaliera Universitaria San Martino-IST di Genova, Genova, Clinica di Medicina Interna 2); Franco Dallegri, Luciano Ottonello, Alessandra Quercioli, Alessandra Barreca (Università di Genova, Genova, Medicina Interna 1); Maria Beatrice Secchi, Davide Ghelfi (Ospedale Bassini di Cinisello Balsamo, Milano, Divisione Medicina); Wu Sheng Chin, Laura Carassale, Silvia Caporotundo (Ospedale Bassini, Cinisello Balsamo, Milano, Unità Operativa di Geriatria); Luigi Anastasio, Lucia Sofia, Maria Carbone (Ospedale Civile Jazzolino di Vibo Valentia, Vibo Valentia, Medicina interna); Giancarlo Traisci, Lucrezia De Feudis, Silvia Di Carlo (Ospedale Civile Santo Spirito di Pescara, Pescara, Medicina Interna 2); Giovanni Davì, Maria Teresa Guagnano, Simona Sestili (Ospedale Clinicizzato SS. Annunziata, Chieti, Clinica Medica); Elisabetta Bergami, Emanuela Rizzioli (Ospedale del Delta, Lagosanto, Ferrara, Medicina Interna); Carlo Cagnoni, Luca Bertone, Antonio Manucra (Ospedale di
Table 6

Multivariate analysis of the association between variables and 3-month mortality.

\begin{tabular}{llc}
\hline & Odds ratio (C.I. 95\%) & $\mathrm{p}$ \\
\hline Age & $1.05(1.01-1.09)$ & 0.004 \\
Male gender & $2.12(1.23-3.60)$ & 0.006 \\
Barthel Index $\leq 40$ & $4.98(2.70-8.90)$ & $<0.0001$ \\
Systolic blood pressure & $0.98(0.97-0.99)$ & 0.01 \\
Chronic kidney disease & $1.49(0.87-2.60)$ & 0.14 \\
Malignancy & $3.58(1.28-4.11)$ & $<0.0001$ \\
\hline
\end{tabular}

The model reports only variables with $\mathrm{p}<0.2$; see Statistical analysis Section.

Bobbio, Piacenza, Unità Operativa Medicina e Primo Soccorso); Alberto Buratti, Tiziana Tognin, Nicola Lucio Liberato (Azienda Ospedaliera della Provincia di Pavia, Ospedale di Casorate Primo, Pavia, Medicina Interna); Giordano Bernasconi, Barbara Nardo (Ospedale di Circolo di Busto Arsizio, Varese, Medicina I); Giovanni Battista Bianchi, Sabrina Giaquinto Ospedale "SS Gerosa e Capitanio" di Lovere, Bergamo, Unità Operativa Complessa di Medicina Generale, Azienda Ospedaliera "Bolognini" di Seriate, Bergamo; Giampiero Benetti, Michela Quagliolo, Giuseppe Riccardo Centenaro (Ospedale di Melegnano, Vizzolo Predabissi, Melegnano, Medicina 1); Francesco Purrello, Antonino Di Pino, Salvatore Piro (Ospedale Garibaldi Nesima, Catania, Unità Operativa Complessa di Medicina Interna); Gerardo Mancuso, Daniela Calipari, Mosè Bartone, Francesco Gullo (Ospedale Giovanni Paolo II Lamezia Terme, Catanzaro, Unità Operativa Complessa Medicina Interna); Michele Cortellaro, Marina Magenta, Francesca Perego; Maria Rachele Meroni (Ospedale Luigi Sacco, Milano, Medicina 3); Marco Cicardi, Antonio Gidaro Marina Magenta (Ospedale Luigi Sacco, Milano, Medicina II); Andrea Sacco, Antonio Bonelli, Gaetano Dentamaro (Ospedale Madonna delle Grazie, Matera, Medicina); Renzo Rozzini, Lina Falanga, Alessandro Giordano (Ospedale Poliambulanza, Brescia, Medicina Interna e Geriatria); Paolo Cavallo Perin, Bartolomeo Lorenzati, Gabriella Gruden, Graziella Bruno (Dipartimento di Scienze Mediche, Università di Torino, Città della Scienza e della Salute, Torino, Medicina 3); Giuseppe Montrucchio, Elisabetta Greco, Pietro Tizzani (Dipartimento di Scienze Mediche, Università di Torino, Città della Scienza e della Salute, Torino, Medicina Interna 5); Giacomo Fera, Maria Loreta Di Luca, Donatella Renna (Ospedale San Giacomo di Monopoli, Bari, Unità Operativa Medicina Interna); Antonio Perciccante, Alessia Coralli (Ospedale San GiovanniDecollato-Andisilla, Civita Castellana Medicina); Rodolfo Tassara, Deborah Melis, Lara Rebella (Ospedale San Paolo, Savona, Medicina I); Giorgio Menardo, Stefania Bottone, Elsa Sferrazzo (Ospedale San Paolo, Savona, Medicina Interna e Gastroenterologia); Claudio Ferri, Rinaldo Striuli, Rosa Scipioni (Ospedale San Salvatore, L'Aquila, Medicina Interna Universitaria); Raffaella Salmi, Piergiorgio Gaudenzi, Susanna Gamberini, Franco Ricci (Azienda Ospedaliera-Universitaria S. Anna, Ferrara, Unità Operativa di Medicina Ospedaliera II); Cosimo Morabito, Roberto Fava (Ospedale Scillesi d'America, Scilla Medicina); Andrea Semplicini, Lucia Gottardo (Ospedale SS. Giovanni e Paolo, Venezia, Medicina Interna 1); Giuseppe Delitala, Stefano Carta, Sebastiana Atzori (Ospedale Universitario Policlinico di Sassari, Sassari, Clinica Medica); Gianluigi Vendemiale, Gaetano Serviddio, Roberta Forlano (Ospedali Riuniti di Foggia, Foggia, Medicina Interna Universitaria); Luigi Bolondi, Leonardo Rasciti, Ilaria Serio (Policlinico Sant'OrsolaMalpighi, Bologna, Unità Operativa Complessa Medicina Interna); Cesare Masala, Antonio Mammarella, Valeria Raparelli (Policlinico Umberto I, Roma, Medicina Interna D); Filippo Rossi Fanelli, Massimo Delfino, Antonio Amoroso (Policlinico Umberto I, Roma, Medicina Interna H); Francesco Violi, Stefania Basili, Ludovica Perri (Policlinico Umberto I, Roma, Prima Clinica Medica); Pietro Serra, Vincenzo Fontana, Marco 
Falcone (Policlinico Umberto I, Roma, Terza Clinica Medica); Raffaele Landolfi, Antonio Grieco, Antonella Gallo (Policlinico Universitario A. Gemelli, Roma, Clinica Medica); Giuseppe Zuccalà, Francesco Franceschi, Guido De Marco, Cordischi Chiara, Sabbatini Marta (Policlinico Universitario A. Gemelli, Roma, Roma, Unità Operativa Complessa Medicina d'Urgenza e Pronto Soccorso); Martino Bellusci. Donatella Setti, Filippo Pedrazzoli (Presidio Ospedaliero Alto Garda e Ledro, Ospedale di Arco, Trento, Unità Operativa di Medicina Interna Urgenza/Emergenza); Giuseppe Romanelli, Caterina Pirali, Claudia Amolini (Spedali Civili di Brescia, Brescia, Geriatria); Enrico Agabiti Rosei, Damiano Rizzoni, Luana Castoldi (Spedali Civili di Brescia, Brescia, Seconda Medicina); Antonio Picardi, Umberto Vespasiani Gentilucci, Chiara Mazzarelli, Paolo Gallo (Università Campus Bio-Medico, Roma, Medicina Clinica-Epatologia); Luigina Guasti, Luana Castiglioni, Andrea Maresca, Alessandro Squizzato, Sara Contini, Marta Molaro (Università degli Studi dell'Insubria, Ospedale di Circolo e Fondazione Macchi, Varese, Medicina Interna I); Giorgio Annoni, Maurizio Corsi, Sara Zazzetta (Università degli studi di Milano-Bicocca Ospedale S. Gerardo, Monza, Unità Operativa di Geriatria); Marco Bertolotti, Chiara Mussi, Roberto Scotto, Maria Alice Ferri, Francesca Veltri (Università di Modena e Reggio Emilia, AUSL di Modena, Modena, Nuovo Ospedale Civile, Unità Operativa di Geriatria); Franco Arturi, Elena Succurro, Giorgio Sesti, Umberto Gualtieri (Università degli Studi Magna Grecia, Policlinico Mater Domini, Catanzaro, Unità Operativa Complessa di Medicina Interna); Francesco Perticone, Angela Sciacqua, Michele Quero, Chiara Bagnato (Università Magna Grecia Policlinico Mater Domini, Catanzaro, Unità Operativa Malattie Cardiovascolari Geriatriche); Paola Loria, Maria Angela Becchi, Gianfranco Martucci, Alessandra Fantuzzi, Mauro Maurantonio (Università di Modena e Reggio Emilia, Medicina Metabolica-NOCSAE, Baggiovara, Modena); Roberto Corinaldesi, Roberto De Giorgio, Mauro Serra, Valentina Grasso, Eugenio Ruggeri, Lorenzo Mauro Carozza, Fabio Pignatti (Dipartimento di Scienze Mediche e Chirurgiche, Unità Operativa di Medicina Interna, Università degli Studi di Bologna/ Azienda Ospedaliero-Universitaria S. Orsola-Malpighi, Bologna).

\section{References}

[1] Cohen JE. Human population: the next half century. Science 2003;302(5648):1172-5.

[2] Lunenfeld B, Stratton P. The clinical consequences of an ageing world and preventive strategies. Best Pract Res Clin Obstet Gynaecol 2013;27:643-59.

[3] Global population ageing: peril or promise? World Economic Forum; 2012

[4] Nardi R, Scanelli G, Corrao S, Iori I, Mathieu G, Cataldi Amatrian R. Co-morbidity does not reflect complexity in internal medicine patients. Eur J Intern Med 2007;18(5):359-68

[5] Marengoni A, Angleman S, Melis R, Mangialasche F, Karp A, Garmen A, et al. Aging with multimorbidity: a systematic review of the literature. Ageing Res Rev 2011;10(4):430-9.

[6] Wu SY, Green A. Projection of chronic illness prevalence and cost inflation. Washington DC: RAND Health; 2000.

[7] Wolff JL, Starfield B, Anderson G. Prevalence, expenditures and complications of multiple chronic conditions in the elderly. Arch Intern Med 2002;162:2269-76.

[8] Van den Akker M, Buntinx F, Metsemakers JF, Roos S, Knottnerus JA. Multimorbidity in general practice: prevalence, incidence, and co-occurring chronic determinants and recurrent diseases. J Clin Epidemiol 1998;51:367-75.

[9] Maas A, van der Schouw YT, Regitz-Zagrosek V, Swahn E, Appelman YE, Pasterkamp G, et al. Eur Heart J 2011:32:1362-8.

[10] Mosca L, Benjamin EJ, Berra K, Bezanson JL, Dolor RJ, Lloyd-Jones DM, et al Effectiveness-based guidelines for the prevention of cardiovascular disease in women 2011 update: a guideline from the American Heart Association. Circulation 2011:123:1243-62.

[11] Kessler RC. Epidemiology of women and depression. J Affect Disord 2003;74:5-13.

[12] Noble RE. Depression in women. Metab Clin Exp 2005;54:49-52.

[13] Mielke MM, Vemuri P, Rocca WA. Clinical epidemiology of Alzheimer's disease: assessing sex and gender differences. Clin Epidemiol 2014;6:37-48.

[14] Orfila F, Ferrer M, Lamarca R, Tebe C, Domingo-Salvany A, Alonso J. Gender differences in health-related quality of life among the elderly: the role of objective functional capacity and chronic conditions. Soc Sci Med 2006:63:2367-80.

[15] Bowling A, Seetai S, Morris R, Ebrahim S. Quality of life among older people with poor functioning. The influence of perceived control over life. Age Ageing 2007;36:310-5
[16] Nobili A, Licata G, Salerno F, Pasina L, Tettamanti M, Franchi C, et al. SIMI investigators. Polypharmacy, length of hospital stay, and in-hospital mortality among elderly patients in internal medicine wards. The REPOSI study. Eur J Clin Pharmacol 2011;67:507-19.

[17] International classification of diseases ninth revision. www.who.int/classification/ icd/en.

[18] Katzman R, Brown T, Fuld P, Peck A, Schechter R, Schimmel H. Validation of a short orientation-memory-concentration test of cognitive impairment. Am J Psychiatry 1983:140:734-9.

[19] Hickie C, Snowdon J. Depression scales for the elderly: GDS, Gilleard, Zung. Clin Gerontol 1987;6:51-3.

[20] Mahoney FI, Barthel DW. Functional evaluation: the Barthel Index. Md State Med J 1965;14:61-5.

[21] Linn BS, Linn MW, Gurel L. Cumulative illness rating scale. J Am Geriatr Soc 1968;16:622-6.

[22] Gupta A, Lampropulos JF, Bikdeli B, Mody P, Chen R, Kulkarni VT, et al. Most important outcomes research papers on cardiovascular disease in women. Circ Cardiovasc Qual Outcomes 2013;6:e1-7.

[23] Gruneir A, Forrester J, Camacho X, Gill SS, Bronskill SE. Gender differences in home care clients and admission to long-term care in Ontario, Canada: a populationbased retrospective cohort study. BMC Geriatr 2013;13:48.

[24] Hosmer DW, Lemeshow S. Applied logistic regression. New York: J Wiley; 2002.

[25] Penninx BW, van Tilburg T, Kriegsman DM, Deeg DJ, Boeke AJ, van Eijk JT. Effects of social support and personal coping resources on mortality in older age: the longitudinal aging study Amsterdam. Am J Epidemiol 1997;146:510-9.

[26] Hawkley LC, Masi CM, Berry JD, Cacioppo JT. Loneliness is a unique predictor of age-related differences in systolic blood pressure. Psychol Aging 2006;21:152-64.

[27] Thurston RC, Kubzansky LD. Women, loneliness, and incident coronary heart disease. Psychosom Med 2009;71:836-42.

[28] Musselman DL, Evans DL, Nemeroff CB. The relationship of depression to cardiovascular disease: epidemiology, biology, and treatment. Arch Gen Psychiatry 1998;55:580-92.

[29] Wassertheil-Smoller S, Shumaker S, Ockene J, Talavera GA, Greenland P, Cochrane B, et al. Depression and cardiovascular sequelae in post-menopausal women. The Women's Health Initiative (WHI). Arch Intern Med 2004:164:289-98.

[30] Shimbo D, Chaplin W, Crossman D, Haas D, Davidson KW. Role of depression and inflammation in incident coronary heart disease events. Am J Cardiol 2005;96:1016-21.

[31] Van der Kooy $\mathrm{K}$, van Hout $\mathrm{H}$, Marwijk $\mathrm{H}$, Marten $\mathrm{H}$, Stehouwer $\mathrm{C}$, Beekman A. Depression and the risk for cardiovascular diseases: systematic review and meta analysis. Int J Geriatr Psychiatry 2007;22:613-26.

[32] Kivimäki M, Hamer M, Batty GD, Geddes JR, Tabak AG, Penttì J, et al. Antidepressant medication use, weight gain and risk of type 2 diabetes mellitus: a population-based study. Diabetes Care 2010;33:2611-6.

[33] Pan A, Okereke OI, Sun QI, Lagroscino G, Manson JE, Willett WC, et al. Depression and incident stroke in women. Stroke 2011:42:2770-5.

[34] Caso V, Santalucia P, Pezzella FR. Depression and incident stroke in women. Womens Health 2012;8:1-3.

[35] Seifert CL, Poppert H, Sander D, Feurer R, Etgen T, Ander K-H, et al. Depressive symptoms and the risk of ischemic stroke in the elderly-influence of age and sex. PLoS One 2012;7:1-6.

[36] Whang W, Kubzansky LD, Kawachi I, Rexrode KM, Kroenke CH, Glynn RJ, et al. Depression and risk of sudden death and coronary heart disease in women: results from the Nurse's Health Initiative Study. J Am Coll Cardiol 2009;53:950-8.

[37] O'Donnell MJ, Xavier D, Liu L, Zhang H, Chin SL, Rao-Melacini P, et al. Risk factors for ischaemic and intracerebral haemorrhagic stroke in 22 countries (the INTERSTROKE study): a case-control study. Lancet 2010;376:112-23.

[38] Verdelho A, Ferro JM. Late onset depressive symptoms can be a marker of cerebral vascular pathology. J Neurol Neurosurg Psychiatry 2008;79:977.

[39] Weeke P, Jensen A, Folke F, Gislason GH, Olesen JB, Andersson C, et al. Antidepressant use and risk of out-of-hospital cardiac arrest: a nationwide case-time-control study. Clin Pharmacol Ther 2012;92:72-9.

[40] Smoller JW, Allison M, Cochrane BB, Curb JD, Perlis RH, Robinson JG, et al. Antidepressant use and risk of incident cardiovascular morbidity and mortality among postmenopausal women in the Women's Health Initiative Study. Arch Intern Med 2009;169:2128-39.

[41] Nahid AA, Bugami MAI, Loy-English I. Gender differences in dementia risk factor. Gend Med 2007;4:120-9.

[42] Dìez-Manglano J, de Escalante Yangüela B, Garcìa-Arilla Calvo E, Ubis Diez E, Munilla Lòpez E, on behalf of the PLUPAR study researchers, et al. Differential characteristics in polypathological inpatients in internal medicine departments and acute geriatric units: the PLUPAR study. Eur J Intern Med 2013;24:767-71.

[43] Fried LP, Bandeen-Roche K, Kasper JD. Guralnik JM for the Women's Health and Aging Study collaborative research group. J Clin Epidemiol 1999;52:27-37.

[44] Seguin R, LaMonte M, Tinker L, Liu J, Woods N, Michael YL, et al. Sedentary behavior and physical function decline in older women: finding from the Women's Health Initiative. J Aging Res 201210 pp: Artcle ID 271589. Published online May 21, 2012. http://dx.doi.org/10.1155/2012/271589.

[45] Molarius A, Janson S. Self-rated health, chronic diseases, and symptoms among middle-aged and elderly men and women. J Clin Epidemiol 2002;55:364-70.

[46] Cherepanov D, Palta M, Fryback DG, Robert SA. Gender differences in health-related quality-of-life are partly explained by sociodemographic and socioeconomic variation between adult men and women in the US: evidence from four US nationally representative data sets. Qual Life Res 2010;19:1115-24.

[47] Bernabeu-Wittel M, Ollero-Baturone M, Moreno-Gaviño L, Barón-Franco B, Fuertes A, Murcia-Zaragoza J, et al. Eur J Intern Med 2011;22:311-7.

[48] Bushnell C, Reeves MJ, Zhao X, Pan W, Prvu-Bettger J, Zimmer L, et al. Sex differences in quality of life after ischemic stroke. Neurology 2014;82:1-10. 